\title{
Archivos de Cardiología de México

\section{Glenn bidireccional: resultados y análisis de factores de riesgo en una experiencia de 5 años}

\author{
Verónica Becerra ${ }^{a, *}$, Guillermo Moreno $^{a}$, María Althabe ${ }^{\mathrm{a}}$, Ana Lenz ${ }^{\mathrm{a}}$, \\ Ricardo Magliola ${ }^{a}$, Analía Martín ${ }^{b}$, Jorge Barreta ${ }^{c}$, Pablo Ruffa $^{c}$ y José Suárez ${ }^{c}$ \\ a Unidad de Cuidados Intensivos Cardiovasculares, Hospital de Pediatría Prof. Dr. Juan P. Garrahan, Buenos Aires, Argentina \\ b Servicio de Cardiología, Hospital de Pediatría Prof. Dr. Juan P. Garrahan, Buenos Aires, Argentina \\ c Servicio de Cirugía Cardiovascular, Hospital de Pediatría Prof. Dr. Juan P. Garrahan, Buenos Aires, Argentina
}

Recibido el 11 de enero de 2012; aceptado el 21 de enero de 2013

\author{
PALABRAS CLAVE \\ Ventrículo único; \\ Glenn bidireccional; \\ Cardiopatía \\ congénita; \\ Cirugía \\ cardiovascular \\ pediátrica; \\ Argentina
}

\begin{abstract}
Resumen
Objetivo: La anastomosis de Glenn es un procedimiento paliativo en pacientes con ventrículo único. Si bien se asocia a baja morbimortalidad, el objetivo es exponer los resultados en cirugía de Glenn y analizar los factores de riesgo en nuestra población.

Métodos: Estudio retrospectivo de pacientes con cirugía de Glenn entre 2005 y 2009. Se registraron variables demográficas, tipo de ventrículo único, cirugía previa, método de cirugía y evolución en postoperatorio. Se efectuó un análisis estadístico univariado y multivariado para identificar factores de riesgo de mortalidad y/o de internación prolongada (Stata 9.0).

Resultados: Se operaron 101 pacientes, 87 con Glenn bidireccional y 14 con Glenn bilateral. Edad mediana 15 meses (2.5-108), peso $8.5 \mathrm{~kg}(4.2-27)$. El $74 \%$ tenían cirugía previa, en 54 se realizó algún procedimiento asociado al Glenn y en 4 se dejó una fuente adicional de flujo pulmonar. La mediana de internación fue de 8 días (2-97). El 35\% presentó complicaciones postoperatorias. No encontramos asociación entre edad, peso o cirugías previas y mortalidad. Tampoco entre tiempo de bomba y «pinzamiento», procedimientos asociados o Glenn bilateral. La mortalidad fue de $3.9 \%$.

Conclusión: La mortalidad es similar a la de otras series, sin embargo, no se identificaron factores de riesgo independientes asociados con ella. La morbilidad en esta serie fue elevada. () 2012 Instituto Nacional de Cardiología Ignacio Chávez. Publicado por Masson Doyma México S.A. Todos los derechos reservados.
\end{abstract}

Bidirectional Glenn: Outcomes and risk factor analysis in a 5 years' experience

Abstract

Objective: Bidirectional Glenn shunt is a palliative surgical procedure in patients with single ventricle. Although morbimortality in this surgery is low, risk factors have been described.

\section{KEYWORDS}

Single ventricle; Bidirectional Glenn; Congenital heart disease;

\footnotetext{
* Autor para correspondencia: Isidoro Gálvez 3052, Victoria, San Fernando, CP 1644 Buenos Aires, Argentina.

Correo electrónico: draverobecerra@yahoo.com.ar (V. Becerra).
}

1405-9940/\$ - see front matter @ 2012 Instituto Nacional de Cardiología Ignacio Chávez. Publicado por Masson Doyma México S.A. Todos los derechos reservados. http://dx.doi.org/10.1016/j.acmx.2013.01.014 
Pediatric cardiovascular surgery; Argentina
The purpose of this study is to report our outcomes in Glenn surgery, identifying mortality risk factors in our population.

Methods: Retrospective study between 2005 and 2009. Age, weight, previous surgery, surgical procedure, and postoperative condition were analyzed. Results are reported as median and rank, or absolute values and percentage. Uni and multivariate analysis was made to identify risk factors of mortality and/or prolonged hospitalization (Stata 9.0).

Results: One hundred and one patients were operated; 87 bidirectional Glenn and 14 bilateral Glenn. Median age 15 months (2.5-108), median weight $8.5 \mathrm{~kg}$ (4.2-27), and 74\% of them with previous surgery. In 54 patients an associated procedure was performed simultaneously, only in 4 additional pulmonary blood flow was left. Median hospitalization was 8 days (2-97). Thirtyfive percent of the patients presented complications. No association between age, weight, pulmonary hypertension, cardiopulmonary bypass, cross clamping, associated procedures or Glenn bilateral with mortality or morbidity was found. Overall mortality was 3.9\%.

Conclusion: Mortality was similar to other centers, but morbidity in this group of patients is high. No independent risk factors associated with morbidity or mortality were identified.

(c) 2012 Instituto Nacional de Cardiología Ignacio Chávez. Published by Masson Doyma México S.A. All rights reserved.

\section{Introducción}

Desde la década de los 70 los doctores Fontan, Kreutzer, Castañeda y Bjork han desarrollado diversas técnicas quirúrgicas para el tratamiento de las cardiopatías congénitas univentriculares, cuyo objetivo final es crear un sistema hemodinámico en el cual el retorno venoso sistémico es derivado hacia las arterias pulmonares sin pasar por el ventrículo ${ }^{1-5}$. El ventrículo único (VU) funciona como una bomba aspirante (circulación pulmonar pasiva) e impelente (circulación sistémica). Este sistema posee 2 condiciones básicas: resistencias pulmonares bajas y presión de fin de diástole baja del VU. Los pacientes con cirugías de Fontan, Kreutzer y Bjork presentaron complicaciones en el postoperatorio alejado, lo cual motivó estrategias y técnicas quirúrgicas modificadas para mejorar la calidad y sobrevida de estos pacientes ${ }^{6}$. Actualmente hay consenso en realizar la paliación de estas anomalías en etapas, y la anastomosis de Glenn constituye una de las fundamentales dentro de esta estrategia. Consiste en la unión de la vena cava superior (VCS) a la rama pulmonar derecha, en forma terminolateral, y se realiza idealmente alrededor de los 6 meses de edad. $\mathrm{Si}$ bien actualmente la anastomosis de Glenn bidireccional (GB) es un procedimiento que se asocia a baja morbimortalidad, del análisis de la literatura surgen algunos factores de riesgo a considerar como peso, edad, cirugías previas y el Glenn bilateral ${ }^{7-9}$.

El objetivo de este estudio es describir los resultados quirúrgicos en anastomosis de Glenn, y analizar la presencia de factores de riesgo de morbimortalidad en nuestra población.

\section{Métodos}

El Hospital de Pediatría Prof. Dr. Juan P. Garrahan es un centro de alta complejidad, de financiamiento público y atención gratuita, que realiza 700 procedimientos de cirugía cardiovascular por año, y recibe pacientes de todo el país y de la región.

En este estudio de cohorte retrospectiva se incluyeron todos los pacientes sometidos a cirugía de Glenn entre los años 2005 y 2009. Se registraron variables del paciente: sexo, edad, peso, diagnóstico y cirugías cardíacas previas (septectomía, anastomosis, cerclaje y cirugía de Norwood); de la cirugía: tiempo de circulación extracorpórea (CEC), de «pinzamiento» y procedimientos asociados a la anastomosis cavopulmonar (plástica de ramas pulmonares, plástica valvular, septectomía); y del postoperatorio: días de internación, días de ventilación mecánica (VM), complicaciones y mortalidad postoperatoria temprana ( $<30$ días). Se consideró complicación aquella condición que implicara tratamiento adicional y/o prolongación de internación y/o riesgo potencial para la vida del paciente. Las complicaciones se diferenciaron en vinculables al procedimiento de Glenn (parálisis frénica, recurrencial, quilotórax) y vinculables a la cirugía cardíaca en general (infecciones, trombosis, arritmias). Los datos fueron obtenidos de las historias clínicas y de la base de datos del servicio. Por tratarse de un estudio sin intervención no se solicitó consentimiento informado. Se consideraron factores de riesgo la edad $\leq 6$ meses, peso $\leq 5 \mathrm{~kg}$, cirugía previa, procedimientos asociados y Glenn bilateral, por ser los más frecuentemente mencionados en la literatura ${ }^{7-9}$. Se realizó un análisis estadístico bivariado (test de $\mathrm{Chi}^{2}$ y Wilcoxon Rank Sum) para identificar la asociación entre estos factores y la mortalidad y morbilidad. Dada la amplia variedad de diagnósticos de complicaciones, para el análisis de morbilidad se utilizó como variable subrogante la internación $\geq 7$ días. Para identificar factores de riesgo, a fin de eliminar confundidores, se efectuó un análisis multivariado (Stata 9.0). No se realizó cálculo de tamaño muestral por estar predefinido el período de análisis del estudio. Los resultados se expresan en media y desvío estándar o mediana y rango para las variables continuas, y en valores absolutos y porcentaje para las discretas. 


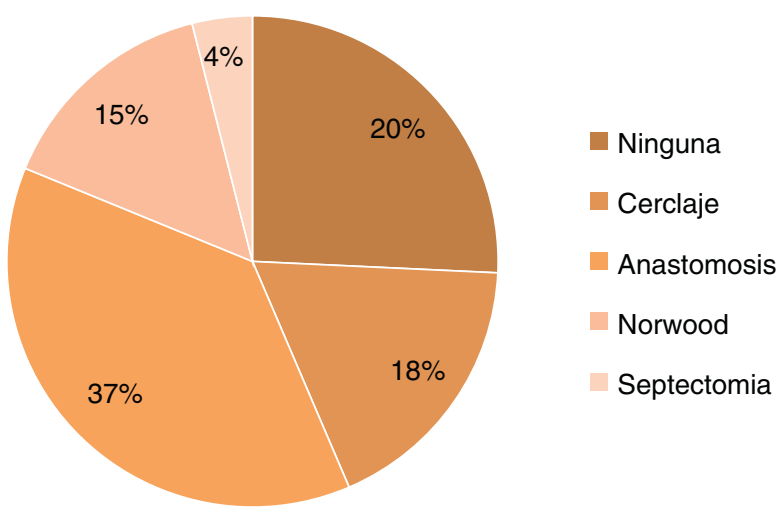

Figura 1 Cirugías previas.

En la actualidad, en nuestra institución el procedimiento de Glenn se considera de elección como fuente de flujo pulmonar en los niños con VU, mayores de 4 meses. La selección de los pacientes se realiza mediante la valoración ecocardiográfica y cateterismo cardíaco para la medición de presiones y el estudio de las ramas pulmonares. Toda estenosis de ramas pulmonares se repara quirúrgicamente siempre que sea posible, o por cateterismo intervencionista cuando no lo es. Se utiliza CEC «stand-by» en todas las cirugías, si bien muchas se realizan con bypass venovenoso; frente a inestabilidad hemodinámica - frecuente en el niño pequeño- es preferible su utilización.

\section{Resultados}

En el período de estudio se realizó cirugía de Glenn a 101 pacientes, en 87 GB y en 14 Glenn bilateral por la presencia de doble sistema cava. La mediana de edad fue de 15 meses (2.5-108), y de peso $8.5 \mathrm{~kg}(4.2-27)$, 61\% varones.

Los diagnósticos más frecuentes fueron atresia tricuspídea (24), VU tipo canal (14), heterotaxias (13), atresia pulmonar con septum intacto (11), hipoplasia de ventrículo izquierdo (7), y en los restantes 32 pacientes, otras formas de VU (tipo derecho, formas complejas de transposición, comunicación interventricular no relacionada, atresia mitral). En nuestra serie, el número de pacientes con corazón izquierdo hipoplásico es muy bajo porque esta enfermedad comenzó a abordarse de manera sistemática en nuestro centro a partir del año 2007. La mayoría de los pacientes (75\%) tenían alguna cirugía previa, la mayor parte, anastomosis sistémico-pulmonar (fig. 1). En relación con el procedimiento quirúrgico, se realizó con bypass venovenoso en $19.8 \%$ y con CEC en el $80.2 \%$ restante, con una duración mediana de $75 \mathrm{~min}$ (28-255). En los casos que requirieron «pinzamiento» aórtico $(n=40)$, la mediana de tiempo fue de 44 minutos (4-150).

De los 42 pacientes que requirieron procedimientos asociados, en más de la mitad el procedimiento fue la plástica de ramas pulmonares (22 pacientes, 52\%), seguida de la plástica de la válvula auriculoventricular en 10 (24\%), septectomía en $6(14 \%)$ y reparación de anomalía de retorno venoso en 4 (9.5\%). En los 12 pacientes restantes (28\%) se realizaron combinaciones de estos procedimientos. En 4 pacientes se dejó una fuente adicional de flujo pulmonar.
Tabla 1 Complicaciones postoperatorias en orden de frecuencia

\begin{tabular}{lrc}
\hline Complicaciones & $\mathrm{n}$ & $\begin{array}{c}\text { Porcentaje/total } \\
\text { de cirugías }\end{array}$ \\
\hline Infección herida quirúrgica & 12 & 11.88 \\
Quilotórax & 8 & 7.92 \\
Arritmias & 6 & 5.94 \\
Neumonia/IRAB & 5 & 4.95 \\
Bacteriemia/sepsis & 4 & 3.96 \\
Parálisis frénica/recurrencial & 4 & 3.96 \\
Neumotórax & 3 & 2.97 \\
Síndrome de VCS & 2 & 1.98 \\
Trombosis venosa & 1 & 0,99 \\
Total & 45 & \\
\hline
\end{tabular}

IRAB: infección respiratoria aguda baja; VCS: vena cava superior.

En relación con la evolución postoperatoria encontramos que, si bien la extubación precoz es la práctica recomendada, 42 pacientes de nuestra serie requirieron VM con una mediana de 2 días (1-83). La injuria pulmonar, el bajo gasto cardíaco y la hipertensión pulmonar se observaron en la mayoría de los pacientes que necesitaron VM por más de $48 \mathrm{~h}$. La mediana de internación en la Unidad de Cuidados Intensivos ( $\mathrm{UCI}$ ) fue de 6 días, y la estancia hospitalaria total, de 8 días (2-97). El $50 \%$ de los pacientes egresaron a su domicilio directamente desde la $\mathrm{UCl}$. La frecuencia de complicaciones fue significativa, 36 pacientes (35\%) presentaron complicaciones, algunos de ellos más de una.

En 15 de ellos el tipo de complicación puede ser atribuible a la técnica quirúrgica (parálisis frénica, recurrencial, quilotórax, síndrome de VCS). La presencia de arritmias (4 pacientes con ritmo nodal resuelto espontáneamente y 2 con bloqueo auriculoventricular, de los cuales uno requirió colocación de marcapasos) se observó en pacientes que requirieron procedimientos asociados, como plástica valvular o septectomía (tabla 1), de esta manera, la morbilidad atribuible a la técnica de anastomosis cavopulmonar fue del $15 \%$.

Se reoperaron 8 pacientes durante la misma internación, ninguno por defecto técnico en la anastomosis cavopulmonar, sino por complicaciones asociadas al procedimiento (2 por plicatura diafragmática, 2 por mediastinitis, uno por marcapasos, otro por anastomosis subclavio-pulmonar, otro por Fontan y un último por pleurodesis por quilotórax).

Un paciente requirió asistencia circulatoria mecánica en el postoperatorio durante $48 \mathrm{~h}$ por disfunción biventricular severa (doble salida de ventrículo derecho, transposición de grandes vasos y estenosis pulmonar, en quien se realizó cirugía de GB y cerclaje interno de arteria pulmonar), con evolución posterior satisfactoria. La mortalidad fue del 3.9\%, 3 pacientes fallecieron por disfunción del Glenn con hipoxemia severa y/o bajo gasto cardíaco, el resto por infección.

En las tablas 2 y 3 se observan los factores de riesgo de mortalidad e internación prolongada. Solo la presencia de cirugía previa se asoció significativamente con internación mayor de 7 días. 
Tabla 2 Factores de riesgo de mortalidad

\begin{tabular}{llll}
\hline Variable & Vivos & Fallecidos & $\mathrm{p}$ \\
\hline Edad $\leq 6 \mathrm{~m}$ & 16 & 0 & 0,37 \\
Peso $\leq 5 \mathrm{~kg}$ & 10 & 0 & 0,49 \\
Glenn bilateral & 14 & 0 & 0,41 \\
Cirugía previa & 71 & 2 & 0,25 \\
Procedimientos asociados & 50 & 4 & 0,06 \\
\hline
\end{tabular}

Tabla 3 Factores de riesgo de internación prolongada ( $\geq 7$ días)

\begin{tabular}{lccl}
\hline Variable & $\begin{array}{l}\text { Internación } \\
\geq 7 \text { días }\end{array}$ & $\begin{array}{l}\text { Internación } \\
<7 \text { días }\end{array}$ & $\mathrm{p}$ \\
\hline Edad $\leq 6 \mathrm{~m}$ & 13 & 3 & 0,06 \\
Peso $\leq 5 \mathrm{~kg}$ & 8 & 2 & 0,17 \\
Glenn bilateral & 6 & 8 & 0,17 \\
Cirugía previa & 48 & 25 & 0,024 \\
Procedimientos & 36 & 18 & 0,11 \\
$\quad$ asociados & & & \\
\hline
\end{tabular}

\section{Discusión}

En nuestra institución, la estrategia del GB, como etapa previa al Fontan en la paliación de pacientes con fisiología univentricular, ha sido utilizada en forma creciente. Esta serie de pacientes constituye la mayor publicada en nuestro país, y los resultados de este período representan la consolidación no solo de una estrategia en el tratamiento del VU, sino de los equipos quirúrgicos y de recuperación, junto con el esfuerzo institucional para aumentar la oferta de turnos quirúrgicos.

Si bien la tendencia actual es realizar la intervención alrededor de los 6 meses o incluso antes, de acuerdo con la situación clínica del paciente, en nuestra serie la edad mediana es mayor (15 meses), y solo 16 de ellos eran $\leq 6$ meses. Las limitaciones para incrementar la oferta de turnos quirúrgicos del sector público frente a una demanda creciente explica esta diferencia ${ }^{10}$.

Como ya han sugerido otros autores ${ }^{11}$, la anastomosis cavopulmonar es la técnica de elección en lactantes cianóticos. La anastomosis subclavio-pulmonar quedaría reservada a neonatos muy sintomáticos que no pueden esperar al GB ${ }^{12}$. Por las razones que hemos mencionado, la mayoría de nuestros pacientes tenían una paliación previa. En la actualidad, a los pacientes a quienes se les realiza una primera paliación se les asigna un turno fijo para la cirugía de Glenn al alta. Dado que la edad no mostró ser un factor de riesgo en nuestra población, esta estrategia no está orientada a modificar los resultados en esta etapa de la reparación, sino a mejorar los resultados de la cirugía de Fontan en el largo plazo, protegiendo al ventrículo de la sobrecarga de volumen y/o presión.

El $50 \%$ de las altas desde la terapia intensiva obedece a una combinación de factores. La mejoría de los resultados permite una rápida descomplejización del paciente, y la falta de disponibilidad de camas en las salas de cuidados intermedios en el hospital han motivado el desarrollo de una estrategia de alta temprana desde la UCI.
En relación con la elevada presencia de complicaciones, cabe destacar que las más frecuentes - las infecciosas, así como el neumotórax o las trombosis asociadas a catéterson eventos que se pueden presentar en cualquier postoperatorio de cirugía cardíaca; no son específicos de la cirugía de Glenn, por lo que la morbilidad atribuible a la técnica de anastomosis cavopulmonar no resulta tan elevada.

A diferencia de otras series publicadas, no encontramos factores de riesgo independientes en nuestra población ${ }^{13}$. Los pacientes con Glenn bilateral, así como los menores de 6 meses, tuvieron un comportamiento similar a los restantes pacientes de la serie. La consolidación de los resultados quirúrgicos y el desarrollo y la implementación de nuevas estrategias en el tratamiento de las cardiopatías congénitas complejas continuarán mejorando la expectativa y calidad de vida de este grupo de pacientes.

\section{Conclusión}

La anastomosis cavopulmonar como cirugía paliativa intermedia previa a la cirugía de Fontan mostró una baja mortalidad, similar a la de otras series publicadas. La morbilidad en este grupo de pacientes fue elevada, pero la atribuible específicamente a la técnica es considerablemente menor. En este estudio no se identificaron factores de riesgo asociados con mayor mortalidad.

\section{Bibliografía}

1. Fontan F, Baudet E. Surgical repair of tricuspid atresia. Thorax. $1971 ; 26: 240-8$.

2. Kreutzer GO, Vargas FJ, Schlichter AJ, Laura JP, Suarez JC, Coronel AR, et al. Atriopulmonary anastomosis. J Thorac Cardiovasc Surg. 1982;83:427-36.

3. Castañeda AR. From Glenn to Fontán. A continuing evolution. Circulation. 1992;86:80-4.

4. Kreutzer G, Galindez E, Bono H, De Palma C, Laura JP. An operation for the correction of tricuspid atresia. J Thorac Cardiovasc Surg. 1973;66:613-21.

5. Bjork VO, Olin CL, Bjarke BB, Thoren CA. Right atrial-right ventricular anastomosis for correction of tricuspid atresia. J Thorac Cardiovasc Surg. 1979;77:452-8.

6. Kreutzer J, Keane JF, Lock JE, Walsh EP, Jonas RA, Castañeda AR, et al. Conversion of modified Fontan procedure to lateral atrial tunnel cavopulmonary anastomosis. J Thorac Cardiovasc Surg. 1996;111:1169-76.

7. Chang AC, Hanley FL, Wernovsky G, Rosenfeld HM, Wessel DL, Jonas RA, et al. Early bidirectional cavopulmonary shunt in young infants. Postoperative course and early results. Circulation. 1993;88 5 Pt 2:I1149-58.

8. Silvilairat S, Pongprot Y, Sittiwangkul R, Woragidpoonpol S, Chuaratanaphong S, Nawarawong W. Factors influencing survival in patients after bidirectional Glenn shunt. Asian Cardiovasc Thorac Ann. 2008;16:381-6.

9. Anderson JB, Beekman RH, Border WL, Kalkwarf HJ, Khoury PR, Uzark $\mathrm{K}$, et al. Lower weight-for-age $\mathrm{z}$ score adversely affects hospital length of stay after the bidirectional Glenn procedure in 100 infants with a single ventricle. J Thorac Cardiovasc Surg. 2009;138:397-404.

10. Magliola R, Laura JP, Capelli H. Situación actual de los niños con cardiopatía congénita en Argentina. Arch Argent Pediatr. 2000;98:130-3.

11. Pridjian AK, Mendelsohn AM, Lupinetti FM, Beekman III RH, Dick II M, Serwer G, et al. Usefulness of the bidirectional Glenn 
procedures stage reconstruction for the functional single ventricle. Am J Cardiol. 1993;71:959-62.

12. Valera MFJ, Caffarena J, Gómez-Ullate JM, Gomez-Plana Usero J, Carrasco JI, Sáez JM, et al. Factores de riesgo en el shunt de Glenn bidireccional como proceder intermedio antes de la corrección de Fontan. Rev Esp Cardiol. 1999;52:903-9.
13. Fernández Pineda L, Cazzaniga M, Villagrá F, Díez Balda JI, Daghero F, Herraiz Sarachaga I, et al. La operación de Glenn bidireccional en 100 casos con cardiopatías congénitas complejas: factores determinantes del resultado quirúrgico. Rev Esp Cardiol. 2001;54:1061-74. 